Georgian Mathematical Journal

Volume 7 (2000), Number 1, 133-154

\title{
ON A NONLOCAL BOUNDARY VALUE PROBLEM FOR SECOND ORDER NONLINEAR SINGULAR DIFFERENTIAL EQUATIONS
}

\author{
A. LOMTATIDZE AND L. MALAGUTI
}

Abstract. Criteria for the existence and uniqueness of a solution of the boundary value problem

$$
u^{\prime \prime}=f\left(t, u, u^{\prime}\right) ; \quad u(a+)=0, \quad u(b-)=\int_{a}^{b} u(s) d \mu(s)
$$

are established, where $f:] a, b\left[\times R^{2} \rightarrow R\right.$ satisfies the local Carathéodory conditions, and $\mu:[a, b] \rightarrow R$ is the function of bounded variation. These criteria apply to the case where the function $f$ has nonintegrable singularities in the first argument at the points $a$ and $b$.

\section{Statement of the Main Results}

Below we shall use the following notations:

$R$ is a set of real numbers.

$L([a, b])$ is the set of functions $p:] a, b[\rightarrow R$ which are Lebesgue integrable on $[a, b]$.

$L_{l o c}(] a, b[)$ is the set of functions $\left.p:\right] a, b[\rightarrow R$ which are Lebesgue integrable on $[a+\varepsilon, b-\varepsilon]$ for arbitrarily small $\varepsilon>0$.

$K_{0}(] a, b\left[\times R^{2}\right)$ is the set of functions $\left.g:\right] a, b\left[\times R^{2} \rightarrow R\right.$ for which the mapping $t \longmapsto g\left(t, x_{1}(t), x_{2}(t)\right)$ is measurable no matter what the continuous functions $\left.x_{i}:\right] a, b[\rightarrow R, i=1,2$, might be.

$\sigma: L_{l o c}(] a, b[) \rightarrow L_{l o c}(] a, b[)$ is the operator defined by the equality

$$
\sigma(p)(t)=\exp \left[\int_{\frac{a+b}{2}}^{t} p(s) d s\right] .
$$

If $\sigma(p) \in L([a, b]), \alpha \in[a, b]$ and $\beta \in] \alpha, b]$, then

$$
\sigma_{\alpha}(p)(t)=\frac{1}{\sigma(p)(t)}\left|\int_{\alpha}^{t} \sigma(p)(s) d s\right|
$$

2000 Mathematics Subject Classification. 34B16.

Key words and phrases. Second order singular equation, nonlocal boundary value problem.

ISSN 1072-947X / \$8.00 / C) Heldermann Verlag www.heldermann.de 


$$
\sigma_{\alpha \beta}(p)(t)=\frac{1}{\sigma(p)(t)} \int_{\alpha}^{t} \sigma(p)(s) d s \cdot \int_{t}^{\beta} \sigma(p)(s) d s .
$$

$u(s+)$ and $u(s-)$ are the limits of the function $u$ at the point $s$ from the right and from the left.

If $\mu:[a, b] \rightarrow R$ is a function of bounded variation, then for each $t \in[a, b]$ by $\mu^{*}(t)$ we denote a total variation of the function $\mu$ on the segment $[a, t]$.

Under the solution of the equation

$$
u^{\prime \prime}=f\left(t, u, u^{\prime}\right)
$$

where $f:] a, b\left[\times R^{2} \rightarrow R\right.$ satisfies the Carathéodory conditions on every compact contained in $] a, b\left[\times R^{2}\right.$, we understand the function $\left.u:\right] a, b[\rightarrow R$ which is absolutely continuous together with its first derivative on every segment from ]$a, b[$, satisfying (1.1) a.e.

In the present paper we concern ourselves with the problem of the existence and uniqueness of a solution of equation (1.1) satisfying the boundary conditions

$$
u(a+)=0, \quad u(b-)=\int_{a}^{b} u(s) d \mu(s),
$$

where $\mu:[a, b] \rightarrow R$ is the function of bounded variation.

The criteria for the unique solvability of the problem in the linear case are contained in $[7,8]$. In the nonlinear case a problem of type (1.1), (1.2) has been considered in [4-6]. However, in these works $\mu$ is assumed to be a piecewise constant function $\left(\mu(t)=0\right.$ for $a \leq t \leq t_{0}$ and $\mu(t)=1$ for $\left.t_{0}<t \leq b\right)$.

The theorems of the existence and uniqueness of a solution of problem (1.1), (1.2) given in the present paper cover the case where $\mu$ is, generally speaking, not piecewise constant, and $f$ is not integrable in the first argument on the segment $[a, b]$, having singularities at the points $t=a$ and $t=b$.

Before we pass to formulating the main results let us introduce the following definitions.

Definition 1.1. We say that a vector-function $\left.\left(p_{1}, p_{2}\right):\right] a, b\left[\rightarrow R^{2}\right.$ belongs to the class $U_{\mu}(] a, b[)$ if

$$
\sigma\left(p_{2}\right), \quad \sigma_{a b}\left(p_{2}\right) p_{1} \in L([a, b]),{ }^{1}
$$

and the solution $u_{1}$ of the singular Cauchy problem

$$
u^{\prime \prime}=p_{1}(t) u+p_{2}(t) u^{\prime} ; \quad u(a+)=0, \quad \lim _{t \rightarrow a+} \frac{u^{\prime}(t)}{\sigma\left(p_{2}\right)(t)}=1^{2}
$$

${ }^{1}$ These conditions are fulfilled if, e.g., $\left|p_{2}(t)\right| \leq \lambda+\frac{\delta}{t-a}+\frac{\delta}{b-t},\left|p_{1}(t)\right| \leq \frac{\lambda}{[(t-a)(b-t)]^{1+\delta}}$, where $\lambda>0,0 \leq \delta<1$.

${ }^{2}$ The unique solvability of this problem has been proved in [2] (see also [3]). 
satisfies the conditions

$$
u_{1}(t)>0 \text { for } a<t<b, \quad u_{1}(b-)>\int_{a}^{b} u_{1}(s) d \mu^{*}(s) .
$$

Definition 1.2. We say that the vector-function $\left.\left(p_{1}, p_{12}, p_{22}\right):\right] a, b\left[\rightarrow R^{3}\right.$ belongs to the class $V_{\mu}(] a, b[)$ if

$$
\begin{gathered}
p_{12}(t) \leq p_{22}(t) \text { for } a<t<b \\
p_{i 2}, p_{1} \in L_{l o c}(] a, b[) \quad i=1,2, \\
\sigma\left(p_{i 2}\right) \in L([a, b]), \quad \sigma_{a b}\left(p_{i 2}\right) p_{1} \in L([a, b]) \quad i=1,2,
\end{gathered}
$$

and $\left(p_{1}, p_{2}\right) \in U_{\mu}(] a, b[)$ no matter what the measurable function $\left.p_{2}:\right] a, b[\rightarrow R$ satisfying the inequalities

$$
p_{12}(t) \leq p_{2}(t) \leq p_{22}(t) \quad \text { for } \quad a<t<b
$$

might be.

Theorem 1.1. On the set $] a, b\left[\times R^{2}\right.$ let the inequalities

$$
\begin{gathered}
{\left[f(t, x, y)-p_{1}(t) x-p_{2}(t, x, y) y\right] \operatorname{sgn} x \geq-p(t),} \\
p_{12}(t) \leq p_{2}(t, x, y) \leq p_{22}(t)
\end{gathered}
$$

be fulfilled, where $p_{2} \in K_{0}(] a, b\left[\times R^{2}\right)$ and

$$
\left(p_{1}, p_{12}, p_{22}\right) \in V_{\mu}(] a, b[) .
$$

Furthermore, let $\sigma_{a b}\left(p_{i 2}\right) p \in L([a, b])(i=1,2)$, and for some point $\left.t_{1} \in\right] a, b[$ let

$$
\begin{gathered}
\left|f(t, x, y)-p_{1}(t) x-p_{2}(t, x, y) y\right| \leq p(t) \\
\text { for } t_{1}<t<b, \quad x \in R, \quad y \in R .
\end{gathered}
$$

Then problem (1.1), (1.2) has at least one solution.

Remark 1.1. Let $\mu$ be nondecreasing, let conditions (1.4)-(1.6) be fulfilled, and $\left(p_{1}, p_{12}, p_{22}\right) \notin V_{\mu}(] a, b[)$. Then there exists a function $f$ satisfying the conditions of Theorem 1.1 for which problem (1.1), (1.2) has no solution.

Remark 1.2. Condition (1.11) can be replaced by the condition

$$
\begin{gathered}
\left|f(t, x, y)-p_{1}(t) x-\widetilde{p}_{2}(t, x, y) y\right| \leq p(t) \\
\text { for } t_{1}<t<b, \quad x \in R, \quad y \in R
\end{gathered}
$$

where $\widetilde{p}_{2} \in K_{0}(] a, b\left[\times R^{2}\right)$ and $p_{12}(t) \leq \widetilde{p}_{2}(t, x, y) \leq p_{2}(t)$ for $\left.(t, x, y) \in\right] t_{1}, b\left[\times R^{2}\right.$. 
As an example let us consider problem (1.1), (1.2) when $\mu$ increases, $\mu(b)-$ $\mu(a)<1$, and

$$
\begin{gathered}
f(t, x, y)=p_{0}(t)+p_{1}(t) x+p_{2}(t) y+p_{3}(t) x^{2 n+1}|y|^{k}, \\
p_{i} \in L_{l o c}(] a, b[), \quad i=\overline{0,3},
\end{gathered}
$$

where $n$ and $k$ are positive integers. Assume that $\lambda>0,0 \leq \delta<1, p_{1}(t) \geq 0$, $p_{3}(t) \geq 0,\left|p_{2}(t)\right| \leq \lambda+\frac{\delta}{t-a}+\frac{\delta}{b-t}$ for $a<t<b$,

$$
\int_{a}^{b}(s-a)(b-s)\left|p_{i}(s)\right| d s<+\infty \quad i=0,1,
$$

and $p_{3}(t) \equiv 0$ in a neighborhood of the point $b$. Taking into account Theorem 1.2 in [8], we obtain from Theorem 1.1 that in this case problem (1.1), (1.2) has at least one solution. As it is seen from the example, the function $f$ may have nonintegrable singularities for $t=a$ and $t=b$.

Corollary 1.1. On the set $] a, b\left[\times R^{2}\right.$ let the inequality

$$
f(t, x, y) \operatorname{sgn} x \geq p_{1}(t)|x|-p_{2}(t)|y|-p(t)
$$

be fulfilled, where $\left(p_{1},-p_{2}, p_{2}\right) \in V_{\mu}(] a, b[)$ and

$$
\sigma_{a b}\left((-1)^{i} p_{2}\right) p \in L([a, b]) \quad i=1,2 .
$$

Furthermore, let for some point $\left.t_{1} \in\right] a, b[$

$$
\begin{gathered}
\left|f(t, x, y)-p_{1}(t) x-p_{2}(t) y\right| \leq p(t) \\
\text { for } t_{1}<t<b, \quad x \in R, \quad y \in R .
\end{gathered}
$$

Then problem (1.1), (1.2) has at least one solution.

Corollary 1.2. Let there exist numbers $\lambda_{i} \in\left[0,1\left[, l_{i} \in\left[0,+\infty\left[, \gamma_{i} \in\right.\right.\right.\right.$ $[0,+\infty[, i=1,2, c \in] a, b[$ and a function $p:] a, b[\rightarrow] 0,+\infty[$ such that

$$
\begin{gathered}
\int_{0}^{+\infty} \frac{d s}{l_{1}+l_{2} s+s^{2}} \geq \frac{(c-a)^{1-\lambda_{1}}}{1-\lambda_{1}}, \quad \int_{\gamma_{1}}^{\gamma_{2}} \frac{d s}{l_{1}+l_{2} s+s^{2}} \geq \frac{(b-c)^{1-\lambda_{2}}}{1-\lambda_{2}}, \\
\int_{\gamma_{1}}^{\gamma_{2}} \frac{s d s}{l_{1}+l_{2} s+s^{2}}<-\ln \left(\mu^{*}(b)-\mu^{*}(a)\right)
\end{gathered}
$$

the function $t \longmapsto(t-a)(b-t) p(t)$ is summable on $[a, b]$, and on the set $] a, b\left[\times R^{2}\right.$ inequality (1.13) is fulfilled, where

$$
\begin{gathered}
p_{1}(t)= \begin{cases}-l_{1}(t-a)^{-2 \lambda_{1}} & \text { for } a<t \leq c \\
-l_{1}(b-t)^{-2 \lambda_{2}} & \text { for } c<t<b\end{cases} \\
p_{2}(t)=\left\{\begin{array}{ll}
l_{2}(t-a)^{-\lambda_{1}}+\lambda_{1}(t-a)^{-1} & \text { for } a<t \leq c \\
l_{2}(b-t)^{-\lambda_{2}}+\lambda_{2}(b-t)^{-1} & \text { for } c<t<b
\end{array} .\right.
\end{gathered}
$$


Moreover, let (1.15) be fulfilled for some point $\left.t_{1} \in\right] a, b[$. Then problem (1.1), (1.2) has at least one solution.

Theorem 1.2. On the set $] a, b\left[\times R^{2}\right.$ let the inequalities

$$
\begin{gathered}
{[f(t, x, y)-f(t, \bar{x}, y)] \operatorname{sgn}(x-\bar{x}) \geq p_{1}(t)|x-\bar{x}|} \\
p_{12}(t)|y-\bar{y}| \leq[f(t, x, y)-f(t, x, \bar{y})] \operatorname{sgn}(y-\bar{y}) \leq p_{22}(t)|y-\bar{y}|
\end{gathered}
$$

be fulfilled, where $\left(p_{1}, p_{12}, p_{22}\right) \in V_{\mu}(] a, b[)$ and

$$
\int_{a}^{b} \sigma\left(p_{i 2}\right)(t)|f(t, 0,0)| d t<+\infty, \quad i=1,2 .
$$

Moreover, let there exist $\left.t_{1} \in\right] a, b\left[\right.$ and a positive function $p \in L_{l o c}(] a, b[)$ such that $\sigma_{a b}\left(p_{i 2}\right) p \in L([a, b]), i=1,2$, and $(1.12)$ is fulfilled, where $\widetilde{p}_{2} \in K_{0}(] a, b\left[\times R^{2}\right)$ and $p_{12}(t) \leq \widetilde{p}_{2}(t, x, y) \leq p_{22}(t)$ for $\left.(t, x, y) \in\right] t_{1}, b\left[\times R^{2}\right.$. Then problem (1.1), (1.2) has one and only one solution.

Remark 1.3. Let $\mu$ be nondecreasing, let conditions (1.4)-(1.6) be fulfilled, and let $\left(p_{1}, p_{12}, p_{22}\right) \notin V_{\mu}(] a, b[)$. Then there exists a function $f$ satisfying the conditions of Theorem 1.2 for which problem (1.1), (1.2) has infinitely many solutions.

Corollary 1.3. On the set $] a, b\left[\times R^{2}\right.$ let the inequality

$$
[f(t, x, y)-f(t, \bar{x}, \bar{y})] \operatorname{sgn}(x-\bar{x}) \geq p_{1}(t)|x-\bar{x}|-p_{2}(t)|y-\bar{y}|
$$

be fulfilled, where $\left(p_{1},-p_{2}, p_{2}\right) \in V_{\mu}(] a, b[)$ and

$$
\int_{a}^{b} \sigma_{a b}\left((-1)^{i} p_{2}\right)(t)|f(t, 0,0)| d t<+\infty, \quad i=1,2 .
$$

Furthermore, let there exist a point $\left.t_{1} \in\right] a, b[$ and a positive function $p \in$ $L_{l o c}(] a, b[)$ such that conditions (1.14) and (1.15) are fulfilled. Then problem (1.1), (1.2) has one and only one solution.

Corollary 1.4. Let there exist numbers $\lambda_{i} \in\left[0,1\left[, l_{i} \in\left[0,+\infty\left[, \gamma_{i} \in\right.\right.\right.\right.$ $\left[0,+\infty[, i=1,2, c \in] a, b\left[, t_{1} \in\right] a, b[\right.$ and a function $p:] a, b[\rightarrow] 0,+\infty[$ such that conditions (1.15)-(1.17) and (1.22) are fulfilled, where $p_{1}$ and $p_{2}$ are the functions defined by equalities (1.18) and (1.19). Moreover, let

$$
\int_{a}^{b}(t-a)(b-t)|f(t, 0,0)| d t<+\infty, \quad \int_{a}^{b}(t-a)(b-t) p(t) d t<+\infty .
$$

Then problem (1.1), (1.2) has one and only one solution. 
Remark 1.4. In the case where $\mu=0$ (see [2]) or $f$ does not depend on the third argument and is integrable in the first argument in the neighborhood of the point $t=b$, then we can neglect condition (1.11) ((1.12)) in Theorems 1.1 and 1.2 .

\section{Some Auxiliary Propositions}

In this paragraph we shall prove some properties of solutions of the equations

$$
u^{\prime \prime}=p_{1}(t) u+p_{2}(t) u^{\prime}+p_{0}(t)
$$

and

$$
u^{\prime \prime}=p_{1}(t) u+p_{2}(t) u^{\prime}
$$

where $p_{i} \in L_{l o c}(] a, b[), i=0,1,2$, and

$$
\sigma\left(p_{2}\right) \in L([a, b]), \quad \sigma_{a b}\left(p_{2}\right) p_{i} \in L([a, b]), \quad i=0,1 .
$$

Note that in this case the well-known Green's theorem is valid (see [8], Theorem 1.1). More precisely, the following lemma is true.

Lemma 2.1. Let condition (2.2) be fulfilled. Then for problem (2.1), (1.2) to be uniquely solvable, it is necessary and sufficient that the corresponding homogeneous problem $\left(2.1_{0}\right)$, (1.2) have only the zero solution. If the latter condition is fulfilled, then there exists the unique Green's function $G$ of problem $\left(2.1_{0}\right),(1.2)$,

$$
\begin{gathered}
G(t, \tau)=\frac{u_{1}(t)}{u_{1}(b-)-\int_{a}^{b} u_{1}(s) d \mu(s)}\left[\int_{\tau}^{b} C(s, \tau) d \mu(s)-C(b-, \tau)\right]+\eta(\tau, t) C(t, \tau) \\
\text { for } \quad a<t, \tau<b
\end{gathered}
$$

where

$$
\eta(t, x)=\left\{\begin{array}{ll}
1 & \text { for } t \leq x \\
0 & \text { for } t>x
\end{array},\right.
$$

$u_{1}$ is the solution of the singular Cauchy problem (1.3), and $C$ is the Cauchy function of equation (2.1 $\left.1_{0}\right)$. Moreover, the solution $u$ of problem (2.1), (1.2) admits the representation

$$
u(t)=\int_{a}^{b} G(t, s) p_{0}(s) d s \quad \text { for } \quad a \leq t \leq b .
$$

Remark 2.1. Taking into account Lemma 2.1 of [5] (see also Lemmas 1.1 and $1.1^{\prime}$ of the monograph [3]), we can easily see that the Green's function $G$ admits the estimates

$$
\begin{aligned}
& |G(t, \tau)| \leq c \sigma_{b}\left(p_{2}\right)(\tau) \int_{a}^{t} \sigma\left(p_{2}\right)(s) d s \text { for } a<t \leq \tau<b \\
& |G(t, \tau)| \leq c \sigma_{a b}\left(p_{2}\right)(\tau) \text { for } a<\tau \leq t<b
\end{aligned}
$$




$$
\left|\frac{\partial}{\partial t} G(t, \tau)\right| \sigma_{a b}\left(p_{2}\right)(t) \leq c \sigma_{a b}\left(p_{2}\right)(\tau) \text { for } a<t, \tau<b,
$$

where $c>0$ is a constant.

\subsection{Properties of the set $U_{\mu}(] a, b[)$.}

Lemma 2.2. Let

$$
\left(p_{1}, p_{2}\right) \in U_{\mu}(] a, b[)
$$

Then $\left(p_{1}, p_{2}\right) \in U_{\mu}(] a_{1}, b[)$, no matter what the point $\left.a_{1} \in\right] a, b[$ might be.

Proof. Let us assume the contrary that the lemma is invalid. Then there exists $\left.a_{1} \in\right] a, b\left[\right.$ such that the solution $u_{0}$ of the Cauchy problem

$$
u^{\prime \prime}=p_{1}(t) u+p_{2}(t) u^{\prime} ; \quad u\left(a_{1}\right)=0, \quad u^{\prime}\left(a_{1}\right)=1,
$$

satisfies the inequalities

$$
u_{0}(t)>0 \text { for } a_{1}<t<b, \quad 0<u_{0}(b-) \leq \int_{a}^{b} u_{0}(s) d \mu^{*}(s) .
$$

Introduce the function

$$
v(t)=u_{1}(t)-\frac{u_{1}(b-)}{u_{0}(b-)} u_{0}(t) \text { for } a_{1} \leq t \leq b,
$$

where $u_{1}$ is the solution of equation $\left(2.1_{0}\right)$ satisfying the initial conditions

$$
u(a+)=0, \quad \lim _{t \rightarrow a+} \frac{u^{\prime}(t)}{\sigma\left(p_{2}\right)(t)}=1 .
$$

Clearly, $v$ is the solution of equation $\left(2.1_{0}\right)$ and

$$
v\left(a_{1}\right)>0, \quad v(b-)=0 .
$$

According to the condition of the lemma and to inequality (2.4) we easily find that

$$
\int_{a_{1}}^{b} v(s) d \mu^{*}(s) \leq \int_{a_{1}}^{b} u_{1}(s) d \mu^{*}(s)-u_{1}(b-) \leq \int_{a}^{b} u_{1}(s) d \mu^{*}(s)-u_{1}(b-)<0 .
$$

Consequently, for some point $\left.t_{0} \in\right] a_{1}, b\left[\right.$ we have $v\left(t_{0}\right)<0$. Taking now into account (2.6), we obtain that the function $v$ has at least two zeros on $\left.] a_{1}, b\right]$, which contradicts the condition of the lemma.

Remark 2.2. If condition (2.3) is fulfilled, then for any $c \in] 0,1[$ we have $\left(p_{1}, p_{2}\right) \in U_{c \mu}(] a, b[)$.

The following lemma is proved analogously.

Lemma 2.3. Let condition (2.3) be fulfilled. Then there exists $\left.\varepsilon_{0} \in\right] 0, \frac{b-a}{2}[$ such that $\left(p_{1}, p_{2}\right) \in U_{\mu}(] a+\varepsilon, b-\varepsilon[)$ for any $\left.\varepsilon \in\right] 0, \varepsilon_{0}[$. 
Remark 2.3. Let condition (2.3) be fulfilled, and let $\varepsilon_{0}$ be the number occurring in Lemma 2.3. For any $\varepsilon \in] 0, \varepsilon_{0}$ [ denote by $u_{\varepsilon}$ the solution of equation $\left(2.1_{0}\right)$ satisfying the conditions

$$
u(a+\varepsilon)=\delta_{1}(\varepsilon), \quad u(b-\varepsilon)=\int_{a+\varepsilon}^{b-\varepsilon} u(s) d \mu(s)+\delta_{2}(\varepsilon) .
$$

Assume that $\delta_{i}(\varepsilon) \rightarrow 0$ for $\varepsilon \rightarrow 0, i=1,2$. Then we can easily see that

$$
\max \left\{\left|u_{\varepsilon}(t)\right|: a+\varepsilon \leq t \leq b+\varepsilon\right\} \rightarrow 0 \text { for } \varepsilon \rightarrow 0 .
$$

Lemma 2.4. Let

$$
\sigma\left(p_{2}\right) \in L([a, b]), \quad \sigma_{a b}\left(p_{2}\right) p_{1} \in L([a, b])
$$

and

$$
\left(p_{1}, p_{2}\right) \notin U_{0}(] a, b[) .
$$

Then for some function $g_{1} \in L_{l o c}(] a, b[)$ satisfying the conditions

$$
g_{1} \geq p_{1} \quad \text { for } \quad a<t<b, \quad \sigma_{a b}\left(p_{2}\right) g_{1} \in L([a, b])
$$

the equation

$$
u^{\prime \prime}=g_{1}(t) u+p_{2}(t) u^{\prime}
$$

has a solution $u$ such that

$$
u(t)>0 \text { for } a<t<b, \quad u(a+)=0, \quad u(b-)=0
$$

moreover, we may assume that $g_{1} \equiv p_{1}$ in some neighborhood of the point $b$.

Proof. Because of (2.8) there exists $a_{1} \in\left[a, b\left[\right.\right.$ such that equation $\left(2.1_{0}\right)$ has the solution $\bar{u}$ satisfying the conditions

$$
\bar{u}(t)>0 \text { for } a_{1}<t<b, \quad \bar{u}\left(a_{1}+\right)=0, \quad \bar{u}(b-)=0 .
$$

If $a_{1}=a$, then we have nothing to prove. Therefore we shall assume that $a_{1}>a$.

Let $v$ be the solution of the boundary value problem

$$
v^{\prime \prime}=\left|p_{1}(t)\right| v+p_{2}(t) v^{\prime} ; \quad v(a+)=0, \quad v(b-)=1 .
$$

Suppose

$$
w(t)=\frac{\bar{u}(t)}{v(t)} \quad \text { for } \quad a_{1}<t<b
$$

Since

$$
w(t)>0 \text { for } a_{1}<t<b, \quad w\left(a_{1}\right)=0, \quad w(b-)=0,
$$

we have

$$
w^{\prime}\left(t^{*}\right)=0
$$


for some point $\left.t^{*} \in\right] a_{1}, b[$, that is,

$$
\frac{\bar{u}\left(t^{*}\right)}{v\left(t^{*}\right)} v^{\prime}\left(t^{*}\right)=\bar{u}^{\prime}\left(t^{*}\right)
$$

Bearing this in mind, we can easily verify that the function

$$
u(t)= \begin{cases}\frac{\bar{u}\left(t^{*}\right)}{v\left(t^{*}\right)} v(t) & \text { for } \quad a \leq t<t^{*} \\ \bar{u}(t) & \text { for } \quad t^{*} \leq t<b\end{cases}
$$

satisfies conditions (2.11) and is the solution of equation (2.10), where

$$
g_{1}(t)= \begin{cases}\left|p_{1}(t)\right| & \text { for } \quad a<t<t^{*} \\ p_{1}(t) & \text { for } t^{*}<t<b\end{cases}
$$

Lemma 2.5. Let $\mu$ be nondecreasing, conditions (2.7) be fulfilled, and

$$
\left(p_{1}, p_{2}\right) \notin U_{\mu}(] a, b[) .
$$

Then for some function $g_{1} \in L_{\text {loc }}(] a, b[)$ satisfying conditions (2.9), equation (2.10) has a solution $u$ such that

$$
u(t)>0 \text { for } a<t<b, \quad u(a+)=0, \quad u(b-)=\int_{a}^{b} u(s) d \mu(s) ;
$$

moreover, we may assume that $g_{1} \equiv p_{1}$ in a neighborhood of the point $b$.

Proof. Owing to (2.12), either (2.8) is fulfilled or $\left(p_{1}, p_{2}\right) \in U_{0}(] a, b[)$, and equation $\left(2.1_{0}\right)$ has the solution $\bar{u}$ satisfying the conditions

$$
\bar{u}(t)>0 \text { for } a<t \leq b, \quad \bar{u}(a+)=0, \quad \bar{u}(b-)<\int_{a}^{b} \bar{u}(s) d \mu(s) .
$$

In the case where (2.8) is fulfilled, by Lemma 2.4 we can assume without loss of generality that equation $\left(2.1_{0}\right)$ has the solution $u$ satisfying conditions (2.11). Thus we may assume that if (2.12) is fulfilled, then equation $\left(2.1_{0}\right)$ has the solution $\bar{u}$ satisfying conditions $(2.14)$.

Let us choose $\left.a_{0} \in\right] a, b[$ such that

$$
\int_{a_{0}}^{b} \sigma_{b}\left(p_{2}\right)(s)\left|p_{1}(s)\right| d s<\frac{1}{2} .
$$

Then (cf., for example, [3], Lemma 1.5 $)$

$$
\left(-\left|p_{1}\right|, p_{2}\right) \in U_{0}(] a_{0}, b[) .
$$

Hence the boundary value problem

$$
u^{\prime \prime}=-\left|p_{1}(t)\right| u+p_{2}(t) u^{\prime} ; \quad u\left(a_{0}\right)=1, \quad u(b-)=1
$$


has the unique solution $u_{0}$. Moreover, $u_{0}(t) \geq 1$ for $a_{0} \leq t \leq b$, since the mapping $t \longmapsto \frac{u_{0}^{\prime}(t)}{\sigma\left(p_{2}\right)(t)}$ does not increase.

Suppose

$$
\begin{gathered}
M=\max \left\{\left|u_{0}(t)\right|: a_{0} \leq t \leq b\right\}+1, \\
v(t, \alpha)=\frac{1}{2 \alpha}\left(\exp \left[\alpha \int_{a}^{t} \sigma\left(p_{2}\right)(s) d s\right]-\exp \left[-\alpha \int_{a}^{t} \sigma\left(p_{2}\right)(s) d s\right]\right) \text { for } a \leq t \leq b .
\end{gathered}
$$

It is easily seen that for some $\alpha_{0}>0$ the inequalities

$$
\sigma\left(p_{2}\right)\left(b_{1}\right) v\left(b_{1}, \alpha_{0}\right)<v^{\prime}\left(b_{1}, \alpha_{0}\right) \int_{b_{1}}^{b} \sigma\left(p_{2}\right)(s) d s
$$

and

$$
2 v\left(a_{1}, \alpha_{0}\right) \int_{a}^{a_{1}} d \mu(s)<v\left(b_{1}, \alpha_{0}\right)
$$

are fulfilled, where $\left.b_{1}=\frac{a_{1}+b}{2}, a_{1} \in\right] a_{0}, b[$ and

$$
2 M \int_{a_{1}}^{b} d \mu(s)<1
$$

Let $u_{1}$ be the solution of the singular Cauchy problem

$$
u^{\prime \prime}=p_{0}(t) u+p_{2}(t) u^{\prime} ; \quad u(a+)=0, \quad \lim _{t \rightarrow a+} \frac{u^{\prime}(t)}{\sigma\left(p_{2}\right)(t)}=1,
$$

where

$$
p_{0}(t)=\left\{\begin{array}{ll}
\alpha_{0}^{2} \sigma\left(p_{2}\right)(t) & \text { for } a<t<b_{1} \\
-\left|p_{1}(t)\right| & \text { for } b_{1}<t<b
\end{array} .\right.
$$

Let us show that

$$
u_{1}(b-)>u_{1}\left(b_{1}\right)
$$

Indeed, since

$$
u_{1}(t)=v\left(t, \alpha_{0}\right) \text { for } \quad a \leq t \leq b_{1},
$$

from (2.20) we find that

$$
\begin{aligned}
u_{1}(t) & =v\left(b_{1}, \alpha_{0}\right)+\frac{v^{\prime}\left(b_{1}, \alpha_{0}\right)}{\sigma\left(p_{2}\right)\left(b_{1}\right)} \int_{b_{1}}^{t} \sigma\left(p_{2}\right)(s) d s \\
& -\int_{t_{1}}^{b} \frac{\int_{\tau}^{t} \sigma\left(p_{2}\right)(s) d s}{\sigma\left(p_{2}\right)(\tau)}\left|p_{1}(\tau)\right| u_{1}(\tau) d \tau \quad \text { for } \quad b_{1} \leq t \leq b,
\end{aligned}
$$


which, by (2.17), implies

$$
u_{1}(t) \leq 2 \frac{v^{\prime}\left(b_{1}, \alpha_{0}\right)}{\sigma\left(p_{2}\right)\left(b_{1}\right)} \int_{b_{1}}^{b} \sigma\left(p_{2}\right)(s) d s \quad \text { for } \quad b_{1} \leq t \leq b .
$$

Because of (2.15) and (2.24) we find from (2.23) that

$$
\begin{gathered}
u_{1}(b-) \geq v\left(b_{1}, \alpha_{0}\right)+\frac{v^{\prime}\left(b_{1}, \alpha_{0}\right)}{\sigma\left(p_{2}\right)\left(b_{1}\right)} \int_{b_{1}}^{b} \sigma\left(p_{2}\right)(s) d s\left[1-2 \int_{b_{1}}^{b} \sigma_{b}\left(p_{2}\right)(s)\left|p_{1}(s)\right| d s\right] \\
>v\left(b_{1}, \alpha_{0}\right)=u_{1}\left(b_{1}\right) .
\end{gathered}
$$

Consequently, (2.20) is fulfilled.

Owing to (2.16) and (2.21), we easily get

$$
u_{1}(t)<M u_{1}(b-) \text { for } a_{1} \leq t \leq b .
$$

Taking into account (2.18), (2.19), (2.22) and (2.25), we have

$$
\begin{aligned}
\int_{a}^{b} u_{1}(s) d \mu(s) & =\int_{a}^{a_{1}} u_{1}(s) d \mu(s)+\int_{a_{1}}^{b} u_{1}(s) d \mu(s) \\
& \leq u_{1}(b-)\left[\int_{a}^{a_{1}} \frac{v\left(s, \alpha_{0}\right)}{u_{1}(b-)} d \mu(s)+M \int_{a_{1}}^{b} d \mu(s)\right] \\
& \leq u_{1}(b-)\left[\frac{1}{2}+\int_{a}^{a_{1}} \frac{v\left(s, \alpha_{0}\right)}{v\left(b_{1}, \alpha_{0}\right)} d \mu(s)\right] \\
& \leq u_{1}(b-)\left[\frac{1}{2}+\frac{v\left(a_{1}, \alpha_{0}\right)}{v\left(b_{1}, \alpha_{0}\right)} \int_{a}^{a_{1}} d \mu(s)\right] \leq u_{1}(b-) .
\end{aligned}
$$

Thus we have shown that the solution $u_{1}$ of problem (2.20) satisfies the conditions

$$
u_{1}(t)>0 \text { for } a<t<b, \quad u_{1}(b-) \geq \int_{a}^{b} u_{1}(s) d \mu(s) .
$$

According to the comparison theorem (see [7], Theorem 1) and Remark 1, the equation

$$
v^{\prime \prime}=\widehat{p}_{0}(t) v+p_{2}(t) v^{\prime}
$$

where

$$
\widehat{p}_{0}=\left\{\begin{array}{ll}
p_{1}(t)+\left|p_{1}(t)\right|+\alpha_{0}^{2} \sigma\left(p_{2}\right)(t) & \text { for } a<t<b_{1} \\
p_{1}(t) & \text { for } b_{1}<t<b
\end{array},\right.
$$

has the solution $v$ satisfying the conditions

$$
v(t)>0 \text { for } a<t<b, \quad v(a+)=0, \quad v(b-)>\int_{a}^{b} v(s) d \mu(s) .
$$


Assume

where

$$
u(t)=\beta_{1} v(t)+\beta_{2} \bar{u}(t) \text { for } a \leq b,
$$

$$
\beta_{1}=\int_{a}^{b} \bar{u}(s) d \mu(s)-\bar{u}(b-)>0, \quad \beta_{2}=v(b-)-\int_{a}^{b} v(s) d \mu(s)>0 .
$$

Clearly, $u$ is the solution of equation (2.10), where

$$
g_{1}(t)=\left\{\begin{array}{ll}
p_{1}(t)+\beta_{1} \frac{\left|p_{1}(t)\right|+\alpha_{0}^{2} \sigma\left(p_{2}\right)(t)}{\beta_{1} v(t)+\beta_{2} \bar{u}(t)} & \text { for } a<t<b_{1} \\
p_{1}(t) & \text { for } b_{1}<t<b
\end{array} ;\right.
$$

moreover, $u$ satisfies conditions (2.13).

\subsection{Properties of the set $V_{\mu}(] a, b[)$.}

Lemma 2.6. Let

$$
\left(p_{1}, p_{12}, p_{22}\right) \in V_{\mu}(] a, b[) .
$$

Then there exists a positive number $r_{0}$ such that no matter what the measurable function $\left.p_{2}:\right] a, b[\rightarrow R$ satisfying the inequalities

$$
p_{12}(t) \leq p_{2}(t) \leq p_{22}(t) \quad \text { for } \quad a<t<b
$$

might be, we have the estimate

$$
\left|u_{1}(b-)-\int_{a}^{b} u_{1}(s) d \mu(s)\right| \geq r_{0}
$$

where $u_{1}$ is the solution of problem $\left(2.1_{0}\right),(2.5)$.

Proof. Let us assume the contrary that the lemma is invalid. Then for any natural $k$ there exists a measurable function $\left.p_{2}^{k}:\right] a, b[\rightarrow R$ satisfying the inequalities

$$
p_{12}(t) \leq p_{2}^{k}(t) \leq p_{22}(t) \text { for } a<t<b
$$

and

$$
\left|u_{1}(b-, k)-\int_{a}^{b} u_{1}(s, k) d \mu(s)\right| \leq \frac{1}{k},
$$

where $u_{1}(\cdot, k)$ is the solution of the singular Cauchy problem

$$
u^{\prime \prime}=p_{1}(t) u+p_{2}^{k}(t) u^{\prime} ; \quad u(a+)=0, \quad \lim _{t \rightarrow a+} \frac{u^{\prime}(t)}{\sigma\left(p_{2}^{k}\right)(t)}=1 .
$$

Introduce the functions

$$
g_{k}(t)=\int_{\frac{a+b}{2}}^{t} p_{2}^{k}(s) d s, \quad k=1,2, \ldots
$$


Because the sequence $\left(g_{k}\right)_{k=1}^{+\infty}$ is uniformly bounded and equicontinuous in ]$a, b\left[^{1}\right.$, we may assume without loss of generality that it converges uniformly in ]$a, b[$. So we can easily see that the function

$$
g(t)=\lim _{k \rightarrow+\infty} g_{k}(t) \text { for } a<t<b
$$

is absolutely continuous in $] a, b\left[\right.$, and its derivative $p_{2}(t)=g^{\prime}(t)$ satisfies inequalities (2.27).

On the other hand, from Lemma 2.1 of [5] (see also [2], the proof of Lemma $2.2)$ it follows that the sequences $\left(u_{1}(\cdot, k)\right)_{k=1}^{+\infty}$ and $\left(u_{1}^{\prime}(\cdot, k)\right)_{k=1}^{+\infty}$ are uniformly bounded on $[a, b]$ and $[a, b-\varepsilon]$, respectively, for sufficiently small $\varepsilon$. Hence, without loss of generality we may assume that the sequences $\left(u_{1}(\cdot, k)\right)_{k=1}^{+\infty}$ and $\left(u_{1}^{\prime}(\cdot, k)\right)_{k=1}^{+\infty}$ uniformly converge in $[a, b[$. Thus, owing to the Krasnosel'skyKrein theorem ([1], p. 328-332), Lemma 2.1' from [5] and estimate (2.28), we see that

$$
u_{1}(t)=\lim _{k \rightarrow+\infty} u_{1}(t, k) \text { for } a<t<b
$$

is the solution of problem $\left(2.1_{0}\right),(2.5)$, and $u_{1}(b-)=\int_{a}^{b} u_{1}(s) d \mu(s)$, which contradicts condition (2.26).

In the sequel we shall need the following notations:

$$
\begin{aligned}
p^{*}(t) & = \begin{cases}p_{12}(t) & \text { for } a<t<\frac{a+b}{2} \\
p_{22}(t) & \text { for } \frac{a+b}{2}<t<b\end{cases} \\
p_{*}(t) & = \begin{cases}p_{22}(t) & \text { for } a<t<\frac{a+b}{2} \\
p_{12}(t) & \text { for } \frac{a+b}{2}<t<b\end{cases} \\
H_{0}(t, \tau) & = \begin{cases}\sigma_{b}\left(p^{*}\right)(\tau) \int_{a}^{t} \sigma\left(p^{*}\right)(s) d s & \text { for } a<t<\tau<b \\
\sigma_{a b}\left(p^{*}\right)(\tau) & \text { for } a<\tau<t<b\end{cases} \\
H_{1}(t, \tau) & =\frac{\sigma_{a b}\left(p^{*}\right)(\tau)}{\sigma_{a b}\left(p_{*}\right)(t)} \text { for } a<t, \tau<b, \\
H(t, \tau) & = \begin{cases}\sigma_{b}\left(p^{*}\right)(\tau) \int_{a}^{t} \sigma\left(p^{*}\right)(s) d s & \text { for } a<t<\tau<b \\
\sigma_{a}\left(p^{*}\right)(\tau) \int_{t}^{b} \sigma\left(p^{*}\right)(s) d s & \text { for } a<\tau<t<b\end{cases}
\end{aligned}
$$

Because of Lemmas 2.1, 2.2 and 2.6, and taking into consideration Lemma 2.1 of [5], we easily see that the lemma below is valid.

Lemma 2.7. Let (2.26) be fulfilled. Then there exists a positive number $r_{1}$ such that no matter what the measurable function $p_{2} \in L_{l o c}(] a, b[)$ satisfying inequalities (2.27) might be, the Green's function $G$ of problem $\left(2.1_{0}\right),(1.2)$ is nonpositive, and

$$
|G(t, \tau)| \leq r_{1} H_{0}(t, \tau) \text { for } a<t, \tau<b
$$

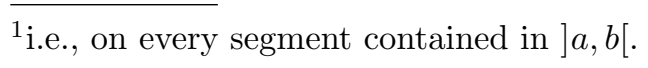




$$
\left|\frac{\partial}{\partial t} G(t, \tau)\right| \leq r_{1} H_{1}(t, \tau) \text { for } a<t, \tau<b .
$$

Lemma 2.8. Let (2.26) be fulfilled. Then there exists a positive number $r$ such that no matter what the functions $g, g_{i} \in L_{l o c}(] a, b[), i=1,2$, satisfying the conditions

$$
\begin{gathered}
\sigma_{a b}\left(p_{i 2}\right) g, \quad \sigma_{a b}\left(p_{i 2}\right) g_{1} \in L([a, b]), \quad i=1,2, \\
g_{1}(t) \geq p_{1}(t), \quad p_{12}(t) \leq g_{2}(t) \leq p_{22}(t) \quad \text { for } \quad a<t<b
\end{gathered}
$$

might be, the boundary value problem

$$
\begin{gathered}
u^{\prime \prime}=g_{1}(t) u+g_{2}(t) u^{\prime}+g(t), \\
u(a+)=0, \quad u(b-)=\int_{a}^{b} u(s) d \mu(s)
\end{gathered}
$$

has the unique solution $u$, and

$$
\left|u^{(i)}(t)\right| \leq r \int_{a}^{b} H_{i}(t, s) \widetilde{g}(s) d s \quad \text { for } a<t<b, \quad i=0,1,
$$

where $\widetilde{g}(t)=\frac{1}{2}(|g(t)|-g(t) \operatorname{sgn} u(t))$ for $a<t<b$.

Proof. Because of conditions (2.26), (2.29) and (2.30) from Theorem 1 of [7] it follows that problem (2.31), (2.32) has the unique solution $u$.

Let $v$ be the solution of the problem

$$
v^{\prime \prime}=p_{1}(t) v+g_{2}(t) v^{\prime}-\widetilde{g}(t) ; \quad v(a+)=0, \quad v(b-)=\int_{a}^{b} v(s) d \mu^{*}(s) .
$$

According to Lemma 2.7, $v(t) \geq 0$ for $a \leq t \leq b$. Let us show that

$$
|u(t)| \leq v(t) \text { for } a \leq t \leq b
$$

Assume the contrary that (2.34) is invalid. Then there exist $t_{1} \in[a, b[$ and $\left.\left.t_{2} \in\right] t_{1}, b\right]$ such that

$$
w(t)=|u(t)|-v(t)>0 \quad \text { for } \quad t_{1}<t<t_{2}
$$

moreover, either

$$
w\left(t_{1}+\right)=0, \quad w\left(t_{2}-\right)=0,
$$

or $t_{1}>a\left(t_{1}=a\right), t_{2}=b$ and

$$
\begin{gathered}
w(t)<0 \text { for } a<t<t_{1}, \quad w\left(t_{1}\right)=0 \\
w(t)>0 \text { for } t_{1}<t \leq b \quad(w(t)>0 \text { for } a<t \leq b) .
\end{gathered}
$$

It is clear that $w$ satisfies the equation

$$
w^{\prime \prime}=p_{1}(t) w+g_{2}(t) w^{\prime}+g_{0}(t),
$$


where

$$
g_{0}(t)=g(t) \operatorname{sgn} u(t)+\widetilde{g}(t)+\left[g_{1}(t)-p_{1}(t)\right]|u(t)| \text { for } a<t<b ;
$$

moreover,

$$
g_{0}(t) \geq 0 \text { for } t_{1}<t<t_{2}
$$

Let (2.36) be fulfilled. Then

$$
w(b-)=|u(b-)|-v(b-) \leq \int_{a}^{b}(|u(s)|-v(s)) d \mu^{*}(s) \leq \int_{t_{1}}^{b} w(s) d \mu^{*}(s) .
$$

Hence $w$ satisfies the conditions

$$
w\left(t_{1}+\right)=0, \quad w(b-)=\int_{t_{1}}^{b} w(s) d\left(\varepsilon \mu^{*}(s)\right),
$$

where $\varepsilon \in] 0,1]$.

According to Lemmas 2.1 and 2.7, Remark 2.2 and condition (2.37), we have

$$
w(t) \leq 0 \text { for } t_{1}<t<b,
$$

which contradicts condition (2.36). Analogously, we can show that (2.35) does not hold either. Thus we have shown that estimate (2.34) holds.

According to Lemmas 2.1 and 2.7, from (2.34) we have that

$$
|u(t)| \leq \widetilde{c_{1}} \int_{a}^{b} H_{0}(t, s) \widetilde{g}(s) d s \quad \text { for } \quad a \leq t \leq b,
$$

where $\widetilde{c_{1}}$ is a positive number not depending on $u$.

Estimate now $\left|u^{\prime}(t)\right|$. Let $\left.t_{1} \in\right] a, b\left[\right.$ be an arbitrary point, where $u^{\prime}\left(t_{1}\right) \neq 0$. For definiteness, we shall assume that $u\left(t_{1}\right) u^{\prime}\left(t_{1}\right) \geq 0$. Then either

$$
u(t) u^{\prime}(t)>0 \text { for } t_{1}<t<b,
$$

or there exists $\left.t_{2} \in\right] t_{1}, b[$ such that

$$
u(t) u^{\prime}(t)>0 \text { for } t_{1}<t<t_{2}, \quad u^{\prime}\left(t_{2}\right)=0 .
$$

First we assume that condition (2.39) is fulfilled. Multiplying both parts of equality $(2.31)$ by $\sigma_{b-\varepsilon}\left(g_{2}\right)(t)$, where $\left.\varepsilon \in\right] 0, b-t_{1}\left[\right.$, and then integrating from $t_{1}$ to $b-\varepsilon$, we obtain

$$
-u^{\prime}\left(t_{1}\right) \sigma_{b-\varepsilon}\left(g_{2}\right)\left(t_{1}\right)+u(b-\varepsilon)-u\left(t_{1}\right)=\int_{t_{1}}^{b-\varepsilon} \sigma_{b-\varepsilon}\left(g_{2}\right)(s)\left[g_{1}(s) u(s)+g(s)\right] d s,
$$

whence, owing to (2.30), we find

$$
\left|u^{\prime}\left(t_{1}\right)\right| \sigma_{b-\varepsilon}\left(g_{2}\right)\left(t_{1}\right) \leq|u(b-\varepsilon)|+\int_{t_{1}}^{b-\varepsilon} \sigma_{b-\varepsilon}\left(g_{2}\right)(s)\left|p_{1}(s)\right||u(s)| d s
$$




$$
+\int_{t_{1}}^{b-\varepsilon} \sigma_{b-\varepsilon}\left(g_{2}\right)(s) \widetilde{g}(s) d s .
$$

As $\varepsilon$ tends to zero, from the last inequality we get

$$
\begin{aligned}
\left|u^{\prime}\left(t_{1}\right)\right| \sigma_{b}\left(g_{2}\right)\left(t_{1}\right) & \leq|u(b-)|+\int_{t_{1}}^{b} \sigma_{b}\left(g_{2}\right)(s)\left|p_{1}(s)\right||u(s)| d s \\
& +\int_{t_{1}}^{b} \sigma_{b}\left(g_{2}\right)(s) \widetilde{g}(s) d s .
\end{aligned}
$$

However

$$
\begin{aligned}
\int_{t_{1}}^{b} \sigma_{b}\left(g_{2}\right)(s) \widetilde{g}(s) d s & \leq\left(\int_{a}^{t_{1}} \sigma\left(g_{2}\right)(s) d s\right)^{-1} \int_{a}^{b} \sigma_{a b}\left(g_{2}\right)(s) \widetilde{g}(s) d s \\
& \leq\left(\int_{a}^{t_{1}} \sigma\left(g_{2}\right)(s) d s\right)^{-1} \int_{a}^{b} \sigma_{a b}\left(p^{*}\right)(s) \widetilde{g}(s) d s .
\end{aligned}
$$

On the other hand, according to (2.38),

$$
|u(t)| \leq \widetilde{c_{1}} \int_{a}^{b} \sigma_{a b}\left(p^{*}\right)(s) \widetilde{g}(s) d s \quad \text { for } \quad a \leq t \leq b .
$$

Therefore from (2.41) we have

$$
\left|u^{\prime}\left(t_{1}\right)\right| \sigma_{a b}\left(g_{2}\right)\left(t_{1}\right) \leq \widetilde{c_{2}} \int_{a}^{b} \sigma_{a b}\left(p^{*}\right)(s) \widetilde{g}(s) d s \quad \text { for } \quad a \leq t \leq b,
$$

where

$$
\widetilde{c_{2}}=\widetilde{c_{1}} \int_{a}^{b}\left[\sigma\left(p^{*}\right)(s)+\sigma_{a b}\left(p^{*}\right)(s)\left|p_{1}(s)\right|\right] d s+1 .
$$

Consequently,

$$
\left|u^{\prime}\left(t_{1}\right)\right| \leq \widetilde{c_{2}} \int_{a}^{b} H_{1}\left(t_{1}, s\right) \widetilde{g}(s) d s .
$$

Similarly, we can show that estimate (2.42) also holds in the case where (2.40) is fulfilled.

Because $t_{1}$ is taken to be arbitrary, we have from (2.42) that (2.33) holds, where $r=\max \left\{\widetilde{c_{1}}, \widetilde{c_{2}}\right\}$.

Lemma 2.9. Let (2.26) be fulfilled and let $\left.t_{1} \in\right] a, b[$. Then there exists $a$ positive number $r_{2}$ such that no matter what the functions $g, g_{i} \in L_{l o c}(] a, b[)$ 
satisfying conditions (2.29), (2.30) and $g_{1} \equiv p_{1}$ might be, the solution $u$ of the boundary value problem (2.31), (2.32) admits the estimate

$$
\begin{gathered}
\left|u(t)-\int_{a}^{b} u(s) d \mu(s)\right| \leq r_{2}\left[1+\int_{a}^{b} \sigma_{a b}\left(p^{*}\right)(s) \widetilde{g}(s) d s\right] \cdot\left[\int_{t}^{b} \sigma\left(p^{*}\right)(s) d s\right. \\
\left.\quad+\int_{t_{1}}^{b} H(t, s)\left(|g(s)|+\left|p_{1}(s)\right|\right) d s\right] \text { for } t_{1} \leq t \leq b,
\end{gathered}
$$

where $\widetilde{g}(t)=\frac{1}{2}(|g(t)|-g(t) \operatorname{sgn} u(t))$ for $a<t<b$.

Proof. Let $r$ be the number occurring in Lemma 2.8 and let $u$ be the solution of problem (2.31), (2.32),

$$
\widetilde{r}_{1}=r \int_{a}^{b} \sigma_{a b}\left(p^{*}\right)(s) \widetilde{g}(s) d s .
$$

Let $v$ be the solution of the boundary value problem

$$
v^{\prime \prime}=p_{1}(t) v+g_{2}(t) v^{\prime}-|g(t)|-\widetilde{r}_{1}\left|p_{1}(t)\right| ; \quad v\left(t_{1}\right)=2 \widetilde{r}_{1}, \quad v(b-)=0 .
$$

By Lemma 2.7 (in the case where $\mu \equiv 0$ ) we have that $v(t) \geq 0$ for $t_{1} \leq t \leq b$. Similarly to that done in proving Lemma 2.8, we can show that

$$
\left|u(t)-\int_{a}^{b} u(s) d \mu(s)\right| \leq v(t) \quad \text { for } \quad t_{1} \leq t \leq b .
$$

Obviously,

$$
v(t)=2 \widetilde{r}_{1} \frac{u_{2}(t)}{u_{2}\left(t_{1}\right)}+\int_{t_{a}}^{t}\left|G_{0}(t, s)\right|\left(\widetilde{r}_{1}\left|p_{1}(s)\right|+|g(s)|\right) d s \text { for } t_{1} \leq t \leq b,
$$

where $G_{0}$ is the Green's function of the problem

$$
v^{\prime \prime}=p_{1}(t) v+g_{2}(t) v^{\prime} ; \quad v\left(t_{1}\right)=0, \quad v(b-)=0,
$$

and $u_{2}$ is the solution of the singular Cauchy problem

$$
u^{\prime \prime}=p_{1}(t) u+g_{2}(t) u^{\prime} ; \quad u(b-)=0, \quad \lim _{t \rightarrow b-} \frac{u^{\prime}(t)}{\sigma\left(g_{2}\right)(t)}=-1 .^{1}
$$

By Lemma 2.7 from (2.45) we find that

$$
v(t) \leq r_{2}\left[1+\int_{a}^{b} \sigma_{a b}\left(p^{*}\right)(s) \widetilde{g}(s) d s\right]\left[\int_{t}^{b} \sigma\left(p^{*}\right)(s) d s\right)
$$

\footnotetext{
${ }^{1}$ The unique solvability of this problem has been proved in [2].
} 


$$
+\int_{t_{1}}^{b} H(t, s)\left(|g(s)|+\left|p_{1}(s)\right| d s\right] \quad \text { for } \quad t_{1} \leq t \leq b,
$$

where $r_{2}$ is a positive number not depending on $u$. This argument and (2.44) result in estimate (2.43).

Finally, let us give one lemma on the solvability of problem (1.1), (1.2).

Lemma 2.10. On the set $] a, b\left[\times R^{2}\right.$ let the conditions

$$
f(t, x, y)=p_{1}(t) x+p_{2}(t) y+g(t, x, y), \quad|g(t, x, y)| \leq g^{*}(t),
$$

be fulfilled, where $\left(p_{1}, p_{2}\right) \in U_{\mu}(] a, b[), \sigma_{a b}\left(p_{2}\right) g^{*} \in L([a, b])$ and $\left.g:\right] a, b\left[\times R^{2} \longmapsto\right.$ $R$ satisfies the local Carathéodory conditions. Then the boundary value problem (1.1), (1.2) has at least one solution.

Proof. Denote by $C\left([a, b] ; R^{2}\right)$ the set of two-dimensional continuous vectorfunctions $x=\left(x_{1}, x_{2}\right):[a, b] \rightarrow R^{2}$ with the norm $\|x\|=\max \left\{\left|x_{1}(t)\right|+\left|x_{2}(t)\right|:\right.$ $a \leq t \leq b\}$.

Let $G_{1}$ be the Green's function of problem $\left(2.1_{0}\right),(1.2)$, and let $G_{2}(t, \tau)=$ $\sigma_{a b}\left(p_{2}\right)(\tau) \frac{\partial}{\partial t} G_{1}(t, \tau)$.

Taking into account Remark 2.1, we can easily see that the operator $F=$ $\left(F_{1}, F_{2}\right)$ defined by the equality

$$
F_{i}\left(x_{1}, x_{2}\right)(t)=\int_{a}^{b} G_{i}(t, s) g\left(s, x_{1}(s), \frac{x_{2}(s)}{\sigma_{a b}\left(p_{2}\right)(s)}\right) d s, \quad i=1,2,
$$

maps continuously the space $C\left([a, b] ; R^{2}\right)$ into its own compact subset. Therefore, owing to Schauder's principle, there exists a vector-function $\left(x_{1}, x_{2}\right) \in$ $C\left([a, b] ; R^{2}\right)$ such that

$$
x_{i}(t)=\int_{a}^{b} G_{i}(t, s) g\left(s, x_{1}(s), \frac{x_{2}(s)}{\sigma_{a b}\left(p_{2}\right)(s)}\right) d s \quad \text { for } \quad a \leq t \leq b, \quad i=1,2 .
$$

From this we find that $x_{2}(t)=x_{1}^{\prime}(t) \sigma_{a b}\left(p_{2}\right)(t)$, and the function $u(t)=x_{1}(t)$ for $a \leq t \leq b$ is the solution of problem (1.1), (1.2).

\section{Proof of the Main Results}

Proof of Theorem 1.1. Let $r, r_{2}, H_{0}, H_{1}$ and $H$ be respectively the numbers and the functions appearing in Lemmas 2.8 and 2.9. Assume that

$$
\begin{aligned}
& \rho_{i}(t)=r \int_{a}^{b} H_{i}(t, s) p(s) d s \text { for } a<t<b, \quad i=0,1 \\
& \rho_{2}(t)=r_{2}\left[1+\int_{a}^{b} \sigma_{a b}\left(p^{*}\right)(s) p(s) d s\right]
\end{aligned}
$$




$$
\begin{aligned}
& \times\left[\int_{t}^{b} \sigma\left(p^{*}\right)(s) d s+\int_{t_{1}}^{b} H(t, s)\left(p(s)+\left|p_{1}(s)\right|\right) d s\right] \text { for } t_{1}<t<b, \\
& \chi_{i}(t, x)= \begin{cases}1 & \text { for }|x| \leq \rho_{i}(t) \\
2-\frac{|x|+1}{\rho_{i}(t)+1} & \text { for } \rho_{i}(t)<|x|<2 \rho_{i}(t)+1, \quad i=0,1 \\
0 & \text { for }|x| \geq 2 \rho_{i}(t)+1\end{cases} \\
& \psi_{k}(t, x, y)= \begin{cases}\chi_{0}(t, x) \chi_{1}(t, y) & \text { for } t \in\left[a+\frac{b-a}{4 k}, b-\frac{b-a}{4 k}\right] \\
0 & \text { for } t \in] a, a+\frac{b-a}{4 k}[\cup] b-\frac{b-a}{4 k}, b[\end{cases} \\
& k=1,2, \ldots \\
& f_{k}(t, x, y)=\psi_{k}(t, x, y)\left[f(t, x, y)-p_{1}(t) x-p_{12}(t) y\right] \\
& \text { for } a<t<b, \quad x, y \in R, \quad k=1,2, \ldots \text {. }
\end{aligned}
$$

Clearly, $f_{k}^{*}(\cdot)=\sup \left\{\left|f_{k}(\cdot, x, y)\right|:(x, y) \in R^{2}\right\} \in L([a, b])$.

Therefore, by Lemma 2.10 , for any natural $k$ the boundary value problem

$$
u^{\prime \prime}=p_{1}(t) u+p_{12}(t) u^{\prime}+f_{k}(t, x, y), \quad u(a+)=0, \quad u(b-)=\int_{a}^{b} u(s) d \mu(s)
$$

has at least one solution $u_{k}$. On account of $(3.2), u_{k}$ is the solution of the equation

$$
u^{\prime \prime}=p_{1}(t) u+g_{2}(t) u^{\prime}+g(t)
$$

where

$$
g_{2}(t)=p_{12}(t)+\psi_{k}\left(t, u_{k}(t), u_{k}^{\prime}(t)\right)\left[p_{2}\left(t, u_{k}(t), u_{k}^{\prime}(t)\right)-p_{12}(t)\right] \text { for } a<t<b
$$

and

$$
\begin{aligned}
g(t) & =\psi_{k}\left(t, u_{k}(t), u_{k}^{\prime}(t)\right)\left[f\left(t, u_{k}(t), u_{k}^{\prime}(t)\right)-p_{1}(t) u_{k}(t)\right. \\
& \left.-p_{2}\left(t, u_{k}(t), u_{k}^{\prime}(t)\right) u_{k}^{\prime}(t)\right] \quad \text { for } \quad a<t<b .
\end{aligned}
$$

Owing to (1.8), (1.9), (1.11), (3.1) and (3.2) the inequalities

$$
\begin{gathered}
p_{12}(t) \leq g_{2}(t) \leq p_{22}(t) \text { for } a<t<b, \\
\widetilde{g}(t)=\frac{1}{2}\left(|g(t)|-g(t) \operatorname{sgn} u_{k}(t)\right) \leq p(t) \text { for } a<t<b, \\
|g(t)| \leq p(t) \text { for } t_{1}<t<b
\end{gathered}
$$

are fulfilled.

By Lemmas 2.8 and 2.9 and conditions (1.10), (3.6)-(3.8) we have the estimates

$$
\left|u_{k}^{(i)}(t)\right| \leq \rho_{i}(t) \quad \text { for } \quad a<t<b, \quad i=0,1
$$




$$
\left|u_{k}(t)-\int_{a}^{b} u_{k}(s) d \mu(s)\right| \leq \rho_{2}(t) \quad \text { for } \quad t_{1}<t<b .
$$

On account of (3.1), (3.2), (3.4), (3.5) and (3.9), from (3.3) we obtain that the restriction $u_{k}$ on $\left[a+\frac{b-a}{k}, b-\frac{b-a}{k}\right]$ is the solution of equation (1.1).

It is easily seen that the sequences $\left(u_{k}\right)_{k=1}^{+\infty}$ and $\left(u_{k}^{\prime}\right)_{k=1}^{+\infty}$ are uniformly bounded and equicontinuous in $] a, b[$. Therefore, without loss of generality we can assume that they converge uniformly in $] a, b[$. Obviously,

$$
\lim _{k \rightarrow+\infty} u_{k}(t)=u(t) \text { for } a<t<b
$$

is the solution of equation (1.1). On the other hand, since $\rho_{0}(a+)=0$ and $\rho_{2}(b-)=0$, it follows from (3.9) and (3.10) that $u$ satisfies the boundary conditions (1.2).

On Remark 1.1. Let (1.4)-(1.6) be fulfilled, and let $\left(p_{1}, p_{12}, p_{22}\right) \notin V_{\mu}(] a, b[)$. Then there exists a measurable function $\left.p_{2}:\right] a, b[\rightarrow R$ satisfying inequalities (1.7), and $\left(p_{1}, p_{2}\right) \notin U_{\mu}(] a, b[)$. Let $g_{1}$ be the function chosen by Lemma 2.5, and let $u_{0} \neq 0$ be the solution of problem (2.10), (1.2). The function $f(t, x, y)=$ $g_{1}(t) x+p_{2}(t) y+u_{0}(t)$ satisfies conditions (1.8), (1.11). On the other hand, we can easily see that (cf., for example, [7], Theorem 1) the equation $u^{\prime \prime}=$ $g_{1}(t) u+p_{2}(t) u^{\prime}+u_{0}(t)$ has no solution satisfying the boundary conditions (1.2).

Proof of Corollary 1.1. It is easy to see that if (1.13) and (1.15) are fulfilled, then (1.8) and (1.12) are also fulfilled, where

$$
\begin{aligned}
& -p_{12}(t)=p_{22}(t)=p_{2}(t), \\
& p_{2}(t, x, y)=\left\{\begin{array}{ll}
p_{2}(t) \operatorname{sgn}(x y) & \text { for } x \neq 0 \\
p_{2}(t) & \text { for } x=0
\end{array} \text { for } a<t<b,\right. \\
& \tilde{p}_{2}(t, x, y)=p_{2}(t) \text { for } t_{1}<t<b, \quad x \in R, y \in R \text {. }
\end{aligned}
$$

Consequently, by Theorem 1.1 and Remark 1.2 problem (1.1), (1.2) has at least one solution.

Proof of Theorem 1.2. From (1.20) and (1.21) we have inequalities (1.8) and (1.9), where

$$
\begin{aligned}
p(t) & =|f(t, 0,0)| \quad \text { for } a<t<b, \\
p_{2}(t, x, y) & =\left\{\begin{array}{ll}
\frac{f(t, 0, y)-f(t, 0,0)}{y} & \text { for } y \neq 0 \\
p_{12}(t) & \text { for } y=0
\end{array} \text { for } a<t<b .\right.
\end{aligned}
$$

Therefore, owing to Theorem 1.1 and Remark 1.2, problem (1.1), (1.2) has at least one solution. It remains for us to show that problem (1.1.), (1.2) has one solution at most. Assume the contrary that this problem has two different 
solutions $u_{1}$ and $u_{2}$. Let

$$
w_{1}(t)=u_{1}(t)-u_{2}(t), \quad w_{2}(t)=-w_{1}(t) \text { for } a \leq t \leq b .
$$

It is clear that for some $i \in\{1,2\}$ either

$$
w_{i}(t) \geq 0 \text { for } a<t<b,
$$

or there exist $t_{1} \in\left[a, b\left[, t_{2} \in\right] t_{1}, b[\right.$ such that for $j \neq i$

$$
w_{j}(t)>0 \text { for } t_{1}<t<t_{2}, \quad w_{j}\left(t_{1}+\right)=0, \quad w_{j}\left(t_{2}\right)=0 .
$$

Suppose first that (3.11) is fulfilled. Without loss of generality we assume that $i=1$. Then on account of (1.20) and (1.21) we have

$$
w^{\prime \prime}=p_{1}(t) w+p_{2}(t) w^{\prime}+h(t) \quad \text { for } \quad a<t<b
$$

where

$$
p_{2}(t)=\left\{\begin{array}{ll}
\frac{f\left(t, u_{2}(t), u_{1}^{\prime}(t)\right)-f\left(t, u_{2}(t), u_{2}^{\prime}(t)\right)}{\left.u_{1}^{\prime}(t)-u_{2}^{\prime}(t)\right)} & \text { for } u_{1}^{\prime}(t) \neq u_{2}^{\prime}(t) \\
p_{12}(t) & \text { for } u_{1}^{\prime}(t)=u_{2}^{\prime}(t)
\end{array} \text { for } a<t<b\right.
$$

and $h(t)=w^{\prime \prime}(t)-p_{1}(t) w(t)-p_{2}(t) w^{\prime}(t)$; moreover, $p_{2}$ satisfies inequalities (1.7), and $h$ is non-negative.

Let $\varepsilon_{0}$ be the number occurring in Lemma 2.3. For any $\left.\varepsilon \in\right] 0, \varepsilon_{0}[$ we denote by $u_{\varepsilon}$ the solution of equation $\left(2.1_{0}\right)$ satisfying the boundary conditions

$$
u_{\varepsilon}(a+\varepsilon)=w_{1}(a+\varepsilon), \quad u_{\varepsilon}(b-\varepsilon)=\int_{a+\varepsilon}^{b-\varepsilon} u_{\varepsilon}(s) d \mu(s)+w(b-\varepsilon)-\int_{a+\varepsilon}^{b-\varepsilon} w(s) d \mu(s) .
$$

By Lemmas 2.1, 2.3, 2.7 and Remark 2.3 we have

$$
w(t)=u_{\varepsilon}(t)+\int_{a+\varepsilon}^{b-\varepsilon} G_{\varepsilon}(t, s) h(s) d s \leq u_{\varepsilon}(t) \text { for } a+\varepsilon<t<b-\varepsilon,
$$

where $G_{\varepsilon}$ is the Green's function of the problem

$$
u^{\prime \prime}=p_{1}(t) u+p_{2}(t) u^{\prime} ; \quad u(a+\varepsilon)=0, \quad u(b-\varepsilon)=\int_{a+\varepsilon}^{b-\varepsilon} u(s) d \mu(s)
$$

and

$$
0 \leq w(t) \leq \lim _{\varepsilon \rightarrow 0} u_{\varepsilon}(t)=0 \text { for } a<t<b
$$

The contradiction obtained above proves that (3.11) cannot take place. Similarly, we can show that condition (3.12) cannot take place either. Consequently, problem (1.1), (1.2) one solution at most.

On Remark 1.3. Let $\mu$ be non-decreasing, the conditions (1.4)-(1.6) be fulfilled, and let $\left(p_{1}, p_{12}, p_{22}\right) \notin V_{\mu}(] a, b[)$. Then there exists the measurable function $\left.p_{2}:\right] a, b\left[\rightarrow R\right.$ satisfying inequalities $(1.7)$, and $\left(p_{1}, p_{2}\right) \notin U_{\mu}(] a, b[)$. Let $g_{1}$ be the function chosen by Lemma 2.5. Clearly, the function $g_{1}(t) x+p_{2}(t) y$ satisfies conditions $(1.20),(1.21)$ and $(1.12)$, where $\widetilde{p}_{2}(t, x, y)=p_{2}(t, x, y)=p_{2}(t)$ and 
$p(t) \equiv 0$. On the other hand, the equation $u^{\prime \prime}=g_{1}(t) u+p_{2}(t) u^{\prime}$ has infinitely many solutions satisfying condition (1.2).

Corollaries 1.2 and 1.4 follow respectively from Corollaries 1.1 and 1.3 and Theorem 4 of [7].

\section{REFERENCES}

1. B. P. Demidovich, Lectures in Mathematical Theory of Stability. (Russian) Nauka, Moscow, 1967.

2. I. T. Kiguradze and A. G. Lomtatidze, On certain boundary value problems for second-order linear ordinary differential equations with singularities. $J$. Math. Anal. Appl. 101(1984), 325-347.

3. I. T. Kiguradze and B. L. Shekhter, Singular boundary value problems for second-order differential equations. (Russian) Current Problems in Mathematics, Newest Results 3, 3-103, Itogi Nauki i Tekhniki, Akad. Nauk SSSR, Vsesoyuzn. Inst. Nauchn. i Tekhn. Inform., Moscow, 1987.

4. A. G. Lomtatidze, On one singular three-point boundary value problem. (Russian) Tbilis. Gos. Univ. Inst. Prikl. Mat. Trudy 17(1986), 122-133.

5. A. G. Lomtatidze, On one boundary value problem for linear ordinary differential equations of second order with singularities. (Russian) Differentsial'nye Uravneniya 22, No. 3,(1986), 416-426.

6. A. G. Lomtatidze, To the question on solvability of singular boundary value problems for ordinary differential equations of second order. (Russian) Tbilis. Gos. Univ. Inst. Prikl. Mat. Trudy 22(1987), 135-149.

7. A. G. Lomtatidze, Nonlocal boundary value problem for linear ordinary differential equations of second order. (Russian) Differentsial'nye Uravneniya 31, No. 3, (1995), 446-455.

8. A. Lomtatidze, On a nonlocal boundary value problem for second order linear ordinary differential equations. J. Math. Anal. Appl. 193(1995), 889908.

(Received 17.10.1998)

Authors' addresses:

A. Lomtatidze

Department of Mathematical Analysis

Faculty of Natural Sciences of Masaryk University

Janackovo nam. 2a, 66295 Brno

Czech Republic

E-mail: bacho@math.muni.cz

L. Malaguti

Dipartimento di Matematica

Universita di Modena

Via Campi 213/B, 41100, Modena

Italy

E-mail: malaguti@unimo.it 